\title{
La société civile au service de l'alphabétisation au Maroc
}

Quel engagement pour quels résultats?

Civil society's role in tackling illiteracy in Morocco. What commitment for what results?

La sociedad civil al servicio de la alfabetización en Marruecos. ¿Cuál es el compromiso y para qué resultados?

\section{Sophie Cerbelle et Mohammed Bougroum}

\section{OpenEdition} Journals

Édition électronique

URL : http://journals.openedition.org/ries/2191

DOI : 10.4000/ries.2191

ISSN : 2261-4265

\section{Éditeur}

Centre international d'études pédagogiques

\section{Édition imprimée}

Date de publication : 1 décembre 2011

Pagination : 83-93

ISBN : 978-2-85420-593-0

ISSN : $1254-4590$

Référence électronique

Sophie Cerbelle et Mohammed Bougroum, « La société civile au service de l'alphabétisation au Maroc », Revue internationale d'éducation de Sèvres [En ligne], 58 I décembre 2011, mis en ligne le 01 décembre 2014, consulté le 19 avril 2019. URL : http://journals.openedition.org/ries/2191 ; DOI : $10.4000 /$ ries. 2191 


\title{
La société civile au service de l'alphabétisation au Maroc
}

\section{Quel engagement pour quels résultats?}

\author{
Sophie Cerbelle \\ Mohammed Bougroum
}

Le retard accumulé par le Maroc en termes de scolarisation jusqu'à la fin des années quatre-vingt-dix explique sa position singulière en termes d'analphabétisme. Selon le dernier recensement, près d'un Marocain sur trois est analphabète et c'est le cas de deux femmes sur trois en zone rurale (HCP-RGPH, 2004). Cette situation contraste avec ses performances sur le plan économique. Alors qu'il occupe la $59^{\mathrm{e}}$ place en terme de niveau économique (PIB/habitant), le Maroc se trouve relégué à la $114^{\mathrm{e}}$ place mondiale en matière de développement humain.

La prise de conscience de ce retard a incité le gouvernement marocain, au-delà des efforts consentis pour améliorer très sensiblement l'accès à l'école, à mettre en place une politique d'alphabétisation de masse à destination des adultes (plus de 15 ans) qui ne sont pas - ou peu - allés à l'école.

Ce regain d'intérêt politique pour l'alphabétisation des adultes a été l'un des résultats du débat national sur l'éducation mené à la fin des années 1990 , et qui a conduit à l'élaboration et l'adoption en 2000 de la Charte nationale d'éducation et de formation (CNEF, 2000). Confiée en 1980 à un simple service de la direction sociale du ministère de l'artisanat et des affaires sociales, la lutte contre l'analphabétisme a été intégrée au secteur de l'éducation depuis 2002, par la création d'un secrétariat d'État en charge de l'alphabétisation et de l'éducation non formelle. En 2007, ce secrétariat d'État a été supprimé au bénéfice d'un projet de création d'une agence nationale de lutte contre l'analphabétisme, qui n'a pas encore abouti à ce jour ${ }^{1}$. Le programme affiché est ambitieux : alors que la proportion d'analphabète parmi les adultes est passée de $61 \%$ en 1990 à $43 \%$ en 2004, l'objectif fixé par la CNEF est de ramener cette proportion à $20 \%$ en 2010 avec la perspective de «l'éradication complète » de ce phénomène en 2015 (Cosef, 2000). 
Pour atteindre cet objectif ambitieux, la stratégie adoptée constitue une rupture importante par rapport aux pratiques antérieures, aussi bien sur le plan quantitatif que sur le plan qualitatif. La pratique d'une offre structurée autour d'un programme principal s'adressant à toute la population a été remplacée par une offre plus diversifiée permettant d'augmenter considérablement la capacité d'accueil. Le ministère de l'Éducation nationale (MEN), avec son Programme général, les opérateurs publics (autres ministères publics), les entreprises, et plus encore la société civile ${ }^{2}$ sont aujourd'hui mobilisés dans la lutte contre l'analphabétisme. Hormis les entreprises, l'ensemble des opérateurs propose une offre de formation similaire, basée sur un programme défini au niveau national, avec un volume horaire global annuel de trois cents heures. Des manuels (en deux tomes jusqu'en 2010, puis regroupés en un seul récemment) édités par le département chargé de l'alphabétisation sont chaque année distribués gratuitement à l'ensemble des opérateurs impliqués.

En matière d'alphabétisation des adultes, la société civile, en raison de son poids numérique croissant (entre 35 et $45 \%$ des inscrits annuels), paraît incontournable dans le paysage. Autrement dit, le succès de la politique d'alphabétisation au Maroc dépend en grande partie de l'action des ONG. Pourtant, l'incertitude sur leurs résultats - en comparaison notamment avec les autres acteurs impliqués (éducation nationale, autres ministères) - demeure (Bougroum M. et al., 2006).

Cet article vise à contribuer au débat actuel sur la pertinence de cette stratégie de "faire faire " adoptée par les pouvoirs publics en matière d'alphabétisation. La question n'est pas tant de remettre en cause cette stratégie, qui paraît indispensable dans le contexte d'une politique d'alphabétisation de masse, mais plutôt de dégager les conditions de son succès. En d'autres termes, si l'implication croissante de la société civile constitue une condition nécessaire pour relever le défi quantitatif d'une politique d'alphabétisation de masse, il est pertinent de s'interroger sur les conditions requises pour que cette implication soit un levier d'une alphabétisation de masse de qualité.

Les exemples abondent dans le secteur de l'éducation formelle pour montrer que les politiques efficientes (celles qui permettent l'atteinte de l'objectif au coût le plus bas) ne sont pas connues a priori et ne se révèlent que dans une analyse pragmatique et patiente des résultats obtenus. Ce sont en effet les évaluations conduites ces quinze dernières années dans le domaine de l'enseignement formel, notamment au niveau de l'enseignement primaire, qui ont progressivement permis de dégager un ensemble de connaissances à l'origine de stratégies plus coût efficaces pour le secteur. Il n'existe rien de comparable en matière d'alphabétisation et d'éducation des adultes, alors que la variété des situations d'enseignement, des publics, des contextes et des intervenants rend au

2. La société civile au Maroc désigne les associations non gouvernementales qui œuvrent dans le domaine du développement. 
moins aussi légitime que dans l'enseignement formel une interrogation sur le choix des activités à entreprendre. Cela exige alors de disposer d'informations sur les résultats des actions entreprises.

Après avoir brièvement rappelé la place des ONG dans la stratégie d'alphabétisation, cet article présentera les résultats d'une recherche menée dans la délégation de Marrakech (Cerbelle, 2010) auprès de populations inscrites dans trois différents programmes d'alphabétisation (ONG, opérateurs publics et Programme général). Cette recherche offre l'opportunité, d'une part, d'interroger les résultats obtenus par les ONG en comparaison avec ceux des autres opérateurs et, d'autre part, de mettre en évidence les caractéristiques (individuelles et contextuelles) les plus favorables à l'alphabétisation. Les implications pour l'action publique sont explorées sous forme de remarques de conclusion.

\section{LA PLACE DES ONG DANS LA STRATÉGIE D'ALPHABÉTISATION}

La stratégie d'alphabétisation au Maroc a été élaborée en 2004 par le secrétariat d'État chargé de l'alphabétisation et de l'éducation non formelle et vise à « éradiquer quasi-totalement le phénomène en 2015 »(SECAENF, 2004). La stratégie vise l'intégration d'un million de personnes par an dans le dispositif d'alphabétisation. Pour ce faire, le gouvernement mobilise autour de lui de nombreux partenaires. L'offre d'alphabétisation est structurée autour de quatre principaux programmes à savoir :

- le Programme général (PG) d'alphabétisation : pris en charge par le ministère de l'Éducation nationale et destiné à l'ensemble des populations analphabètes ne pouvant pas bénéficier d'un programme spécifique d'alphabétisation, il bénéficie des moyens du MEN (un corps de formateurs déjà formés et des locaux équipés disponibles sur l'ensemble du territoire);

- le Programme des opérateurs publics (OP), qui regroupe l'ensemble des autres ministères et administrations qui mettent en œuvre des activités d'alphabétisation au profit de leurs personnels ou des populations relevant de leurs attributions, et parmi lesquels on peut citer, entre autres, le ministère de l'Agriculture, du développement rural et des pêches maritimes, le secrétariat d'État à la Jeunesse (désormais élevé au rang de ministère), le ministère des Habous et des affaires islamiques, l'Entraide nationale, l'Artisanat, les forces auxiliaires ;

- le Programme de la société civile : ce programme est ouvert à toutes les associations qui œuvrent dans le développement social et dont le programme d'action intègre une activité d'alphabétisation; toute association mettant en œuvre une activité d'alphabétisation peut bénéficier d'un soutien logistique de la part du département de tutelle (Direction de la lutte contre l'analphabétisme : DLCA) sous forme de dons de manuels, de guides et de matériels didactiques; 
certaines de ces associations signent une convention de partenariat avec la DLCA, ce qui leur ouvre droit à un soutien financier permettant la prise en charge des indemnités versées aux formateurs; en retour, les ONG conventionnées s'engagent à mettre à disposition des salles de cours, du personnel de formation et d'encadrement; le suivi et le contrôle du programme des ONG sont de la responsabilité de la DLCA;

- le Programme du secteur privé : il regroupe les activités d'alphabétisation menées à l'initiative des entreprises, et qui sont généralement réalisées en partenariat avec les associations professionnelles; le financement de ce programme se fait dans le cadre du système des contrats spéciaux de formation gérés par l'Office de la formation professionnelle et de la promotion du travail (OFPPT).

Grâce à la mobilisation de l'ensemble de ces partenaires, les effectifs d'inscrits dans les programmes d'alphabétisation n'ont cessé d'augmenter ces dernières années.

Depuis 1998-1999, les effectifs inscrits chaque année ont quasiment quadruplé, passant ainsi de 181000 personnes en 1998/1999 à 702119 en 2010-2011. Au total, le Maroc aura donc impliqué en matière d'alphabétisation plus de six millions de personnes en treize ans (sachant qu'une très large majorité d'entre elles a été formée ces cinq dernières années).

Tableau $\mathbf{n}^{\circ} 1$

\begin{tabular}{|c|c|c|c|c|c|}
\cline { 2 - 5 } \multicolumn{1}{c|}{} & $\begin{array}{c}\text { Programme } \\
\text { général }\end{array}$ & $\begin{array}{c}\text { Opérateurs } \\
\text { publics }\end{array}$ & ONG & Entreprises & Total \\
\hline $\mathbf{1 9 9 8 - 1 9 9 9}$ & 89500 & 69000 & 21000 & 1500 & 181000 \\
\hline $\mathbf{1 9 9 9 - 2 0 0 0}$ & 81370 & 66180 & 83500 & 2600 & 233650 \\
\hline $\mathbf{2 0 0 0 - 2 0 0 1}$ & 130035 & 62463 & 105490 & 3500 & 301488 \\
\hline $\mathbf{2 0 0 1 - 2 0 0 2}$ & 62000 & 105000 & 217000 & 6000 & 390000 \\
\hline $\mathbf{2 0 0 2 - 2 0 0 3}$ & 45600 & 77120 & 159400 & 4300 & 286420 \\
\hline $\mathbf{2 0 0 3 - 2 0 0 4}$ & 227650 & 67430 & 153220 & 2040 & 450340 \\
\hline $\mathbf{2 0 0 4 - 2 0 0 5}$ & 127340 & 79430 & 259790 & 2650 & 469210 \\
\hline $\mathbf{2 0 0 5 - 2 0 0 6}$ & 179199 & 236880 & 235070 & 4329 & 655478 \\
\hline $\mathbf{2 0 0 6 - 2 0 0 7}$ & 151795 & 242065 & 310492 & 4803 & 709155 \\
\hline $\mathbf{2 0 0 7 - 2 0 0 8}$ & 105649 & 259109 & 284421 & 2084 & 651263 \\
\hline $\mathbf{2 0 0 8 - 2 0 0 9}$ & 81265 & 286790 & 285124 & 3128 & 656307 \\
\hline $\mathbf{2 0 0 9 - 2 0 1 0}$ & 70087 & 294057 & 338940 & 3310 & 706394 \\
\hline $\mathbf{2 0 1 0 - 2 0 1 1}$ & 49530 & 311320 & 339564 & 1705 & 702119 \\
\hline
\end{tabular}

Source : Cerbelle, 2010. 
La comparaison des effectifs fait apparaître une évolution contrastée selon les opérateurs. La forte augmentation constatée relève essentiellement de la mobilisation des ONG, qui ont multiplié leurs effectifs d'inscrits par quatorze depuis 1998. Les années 2000 voient les ONG s'imposer comme acteur majeur de la lutte contre l'analphabétisme, allant jusqu'à prendre en charge $48 \%$ des effectifs inscrits en 2010-2011.

La forte mobilisation de la société civile à partir de la fin des années 1990, avec l'appui volontariste des pouvoirs publics, a été le déclencheur de fortes campagnes de sensibilisation et d'incitation menées par le tissu associatif auprès des populations analphabètes. Pour autant, les montants alloués aux associations semblent relativement faibles et surtout, nombre d'entre elles fonctionnent sans subvention. Selon les données du secrétariat d'État, les ONG subventionnées (qui prennent en charge environ la moitié des effectifs inscrits au sein du tissu associatif) par le gouvernement reçoivent 240 dirhams par apprenant inscrit. Ce montant est alloué - théoriquement - dans une large mesure à la rémunération des formateurs et, plus marginalement, à la prise en charge des éventuelles dépenses de gestion de l'association. Par comparaison, les montants alloués à l'alphabétisation dans le secteur de l'entreprise dépassent largement les sommes octroyées au secteur associatif (en moyenne, 2000 dirhams par bénéficiaire). Au sein du Programme général (éducation nationale), la somme allouée est sensiblement identique à celle perçue par les ONG, mais à la différence de ces dernières, elle ne sert qu'à financer des heures complémentaires versées aux fonctionnaires participant au programme.

Acteurs incontournables de l'alphabétisation, les ONG jouent également un rôle majeur dans le domaine de la consultation des bénéficiaires. Longtemps négligée, la prise en compte des besoins des populations analphabètes fait l'objet aujourd'hui, aux niveaux central et local, d'une attention particulière, afin de rendre le dispositif d'alphabétisation le plus opérationnel possible. Cette consultation, qui doit être assurée au niveau local par les académies régionales d'éducation et de formation ${ }^{3}$ est néanmoins fortement limitée, en raison notamment de ressources budgétaires insuffisantes. Sur le terrain, ce sont donc plutôt les ONG qui assurent le lien entre le local et le central. Leur proximité avec les bénéficiaires leur permet notamment de mettre en avant les attentes et les contraintes de ces derniers. La consultation des bénéficiaires dépend donc largement - pour ne pas dire uniquement - de la qualité et de l'implication du réseau associatif. Cela revient à dire que l'absence d'ONG dans certaines régions, ou leur manque de moyens, met en péril l'action nationale de lutte contre l'analphabétisme.

3. AREF : Les académies régionales d'éducation et de formation sont les organes décentralisés du ministère de l’Éducation nationale. 


\section{L'EFFICACITÉ COMPARÉE DES ONG}

Les résultats qui suivent sont issus d'une recherche qui s'est déroulée en 2007 dans la délégation de Marrakech, sous les auspices de la DLCA, de l'Université de Marrakech et du Bureau multipays de l'Unesco à Rabat. Le dispositif mis en place, alliant démarches qualitatives et quantitatives, a permis de suivre une cohorte de bénéficiaires inscrits auprès du Programme général, de certains opérateurs publics et des ONG.

Les bénéficiaires ont été soumis à un test de positionnement au moment de leur inscription et à deux tests de mesure des apprentissages (en milieu et en fin de programme). Ces deux derniers tests ont été structurés autour des exercices relatifs aux compétences instrumentales en langue arabe (lire/écrire/ compter) et des exercices relatifs à l'utilisation fonctionnelle de ces connaissances dans la vie courante (remplir un formulaire, se repérer sur une carte, faire une liste de courses, etc.).

Cette recherche offre donc l'opportunité, d'une part, de comparer les résultats des ONG à ceux des autres principaux programmes d'alphabétisation et, d'autre part, d'identifier les raisons des écarts de réussite. L'échantillon se compose comme suit :

Tableau $\mathrm{n}^{\circ} 2$

Répartition des bénéficiaires selon les opérateurs

\begin{tabular}{|c|c|c|}
\hline Opérateurs & Sous-opérateurs & Nombre de bénéficiaires \\
\hline Programme général & & 411 \\
\hline \multirow[t]{7}{*}{ Société civile } & ONG 1 & 88 \\
\hline & ONG 2 & 104 \\
\hline & ONG 3 & 77 \\
\hline & ONG 4 & 123 \\
\hline & ONG 5 & 93 \\
\hline & ONG 6 & 31 \\
\hline & Total effectifs société civile & 516 \\
\hline \multirow[t]{4}{*}{ Opérateurs publics } & Opérateur public 1 & 250 \\
\hline & Opérateur public 2 & 138 \\
\hline & Opérateur public 3 & 87 \\
\hline & Total opérateurs publics & 475 \\
\hline Total & & 1402 \\
\hline
\end{tabular}

Source : Cerbelle, 2010. 
À côté de la passation des trois tests, le protocole de recherche comprenait trois questionnaires de contexte, administrés en face à face respectivement à tous les bénéficiaires, tous les formateurs et tous les responsables de programme, et un dispositif d'observation de classes. La collecte de ces données sociodémographiques sur les bénéficiaires était indispensable pour expliquer la dispersion des résultats à l'issue des programmes de formation. Il est particulièrement difficile d'apprécier "l'efficacité " d'un programme si l'on ne contrôle pas par ailleurs le fait que les bénéficiaires auxquels il s'adresse pourraient être différents.

Les analyses descriptives montrent que ces bénéficiaires sont en moyenne plutôt des femmes, qui vivent maritalement (à plus de $65 \%$ ), dont l'âge se situe autour de 41 ans. Un peu plus de la moitié de l'échantillon vit dans des zones urbaines $(54 \%)$. La très grande majorité de bénéficiaires n'a pas (ou peu) été scolarisée ( $86 \%$ des inscrits ne sont jamais allés à l'école, $11 \%$ ont suivi une année de scolarisation, $2 \%$ deux années et à peine $1 \%$ est resté plus de deux ans à l'école). Bien que cela soit théoriquement impossible de réitérer une inscription dans un dispositif d'alphabétisation, environ $25 \%$ des bénéficiaires ont déjà suivi, par le passé, des cours d'alphabétisation. Une très large majorité (plus de $80 \%$ ) déclare être "sans activité professionnelle». Les activités exercées relèvent toutes du secteur informel (artisanat ou travail domestique notamment).

Les analyses selon l'opérateur ne montrent pas de différences significatives dans le public inscrit. Autrement dit, les bénéficiaires inscrits au sein des ONG ne présentent pas de caractéristiques personnelles fondamentalement différentes des bénéficiaires rattachés au programme général et aux opérateurs publics.

Pour analyser les différences d'apprentissage, un score global (puis par discipline arabe/mathématiques ${ }^{4}$ ) est estimé. L'objectif recherché est d'expliquer ce score par les caractéristiques individuelles des bénéficiaires et par les caractéristiques des opérateurs qui les prennent en charge.

Un premier modèle prend en compte uniquement les variables individuelles des bénéficiaires. Ces variables ont un statut particulier, notamment lorsqu'on s'intéresse aux effets de contexte (ceux qui sont sous la responsabilité des planificateurs); elles ont un statut de variable de contrôle permettant d'aboutir à des mesures non biaisées des effets de contexte.

Ce premier modèle indique que les caractéristiques individuelles des bénéficiaires expliquent dans une large mesure les différences d'acquisition observées en fin de cursus. En moyenne, les hommes réussissent souvent mieux

4. Ces scores sont standardisés de manière traditionnelle dans ce genre d'étude, avec une moyenne de 100 et un écart-type de 15. Ce procédé ne modifie pas le classement des bénéficiaires les uns par rapport aux autres mais l'exprime simplement dans des unités différentes. Il a le mérite de placer dans une même échelle les évaluations initiales et finales qui ne répondent pas spontanément à une unité de mesure commune. 
que les femmes, les jeunes sont plus performants que leurs aînés, les bénéficiaires issus du monde urbain ont de meilleurs résultats que ceux du milieu rural et les personnes actives progressent plus que les autres. Le fait d'avoir été scolarisé, comme celui d'avoir suivi par le passé des cours d'alphabétisation, s'accompagne de meilleures progressions.

Un deuxième modèle estimé prend cette fois en compte les caractéristiques individuelles mais également celles des opérateurs. Les écarts observés entre opérateurs sont estimés ici " toutes choses égales par ailleurs ", c'est-à-dire à public comparable en termes de situation familiale, de scolarisation antérieure, de niveau initial. Les résultats montrent que les ONG, qui, rappelons-le, alphabétisent l'essentiel des effectifs annuels, enregistrent de moins bons résultats que les autres opérateurs, quelle que soit la discipline.

Ces écarts entre opérateurs s'expliquent en grande partie par des différences d'efficacité entre formateurs. A priori, comme pour l'analyse des scores des bénéficiaires, il est possible de rechercher, à travers l'estimation de modèles, les caractéristiques des formateurs les plus à même d'expliquer leurs différences d'efficacité. Un ensemble d'indicateurs relatifs aux caractéristiques personnelles et professionnelles des cinquante-six formateurs de notre échantillon a pu être mobilisé grâce à l'utilisation conjointe de questionnaires et d'entretiens.

L'âge et l'expérience des formateurs influent sur les résultats des bénéficiaires (les formateurs les plus âgés et les plus expérimentés favorisent la réussite). En dehors de ces deux caractéristiques personnelles, les analyses montrent que c'est plutôt leur activité pédagogique qui semble déterminante. Les acquisitions des bénéficiaires sont ainsi positivement associées à l'organisation de réunions, à la rédaction de rapport concernant les programmes, et ces deux facteurs témoignent de la rigueur du suivi des activités menées, en conférant à ces dernières une dimension professionnelle. Les formateurs qui imposent des devoirs à leurs bénéficiaires semblent favoriser leur progression. Enfin, les absences des formateurs pénalisent fortement les progressions des bénéficiaires (en moyenne, une journée d'absence entraîne une baisse du niveau d'acquisition de 2,7 points).

$\mathrm{Au}$ sein de notre échantillon, les formateurs investis dans l'alphabétisation auprès des ONG sont recrutés exclusivement dans la population des diplômés chômeurs. Il s'agit donc essentiellement de jeunes (âgés de 29 ans en moyenne) avec des niveaux d'études qui varient entre le baccalauréat (45\%) et la licence (plus de $40 \%$ ) majoritairement dans des cursus littéraire (droit, histoire, littérature, langue) et, dans une moindre mesure, scientifique (biologie) et économique. L'alphabétisation constitue leur principale activité.

Ils se distinguent en cela des formateurs des autres opérateurs pour qui l'alphabétisation représente une occupation supplémentaire. Les formateurs du Programme général sont tous des enseignants du secteur public titulaire du baccalauréat et du concours de l'Éducation nationale, qui assurent des cours 
d'alphabétisation à l'issue de leur journée d'enseignement scolaire. Les formateurs des opérateurs publics sont des travailleurs sociaux, majoritairement titulaires d'une licence en sciences économiques et sociales et dont l'activité d'alphabétisation constitue une activité qui reste marginale. Ils sont, avec les enseignants du MEN, plus âgés et plus expérimentés que les formateurs des ONG, deux des facteurs favorables aux progressions des bénéficiaires.

Une grande partie des formateurs travaillant auprès d'ONG non subventionnées ne perçoit aucune rémunération. Les plus chanceux d'entre eux, qui collaborent avec des ONG financées par le gouvernement, ne sont cependant pas tellement mieux lotis. Leur rémunération s'apparente plutôt à une indemnisation. Les montants alloués sont faibles et le paiement s'effectue bien souvent très longtemps après les prestations. Les résultats ne font d'ailleurs pas état de différence "d'efficacité » entre les formateurs rémunérés et les bénévoles au sein de l'opérateur ONG, sans doute en raison de la faiblesse et du retard des montants alloués. Cette situation contraste avec celle des formateurs du Programme général et des opérateurs publics, qui disposent d'un contrat de travail, d'un salaire (voire de rétribution d'heures supplémentaires pour les activités d'alphabétisation pour les enseignants) et plus généralement d'un statut professionnel.

Cette précarité des formateurs des ONG est à l'origine d'un fort turnover expliquant ainsi leur moindre ancienneté, à peine deux ans contre six années pour le Programme général et quatre pour les opérateurs publics, expérience qui, nous l'avons vu, est propice aux apprentissages. Cette précarité de statut participe également du fort taux d'absentéisme des formateurs constatés au niveau des ONG et du manque d'encadrement dont ils bénéficient (peu de réunions, pas de compte rendu). Les responsables des ONG, qui ne peuvent se montrer exigeants avec leur personnel "sans statut ", organisent très peu de réunions et n'imposent pas la rédaction de comptes rendus.

De fait, le manque de professionnalisation des formateurs des ONG, qui se traduit par l'absence de statut clair, de perspectives d'avenir, d'encadrement et de rétributions financières (c'est finalement l'opérateur le moins onéreux pour le gouvernement, puisqu'une partie du personnel est bénévole) explique, dans une large mesure, les moins bons résultats obtenus en terme d'apprentissage. L'étude, telle qu'elle est présentée, ne mesure cependant pas d'autres externalités positives et possibles de l'alphabétisation (estime de soi des bénéficiaires, intégration au sein d'un réseau social, changement d'attitudes, etc.) qui auraient peut-être pu contrebalancer les résultats sur les seuls apprentissages, finalement insuffisants pour mesurer la «qualité » des programmes. 
Le passage à une alphabétisation de masse au Maroc pose inévitablement la question de la qualité. Cette question est d'autant plus pertinente que ce passage à l'échelle n'a été possible que grâce à la diversification de l'offre par l'entrée en jeu de la société civile, dont la pluralité organique constitue à la fois une source d'opportunité et de risque. L'implication de la société civile dans la politique d'alphabétisation offre une opportunité pour augmenter les capacités d'accueil, avec une flexibilité permettant de tenir compte de la diversité des populations cibles et des contextes d'alphabétisation. En même temps, cette flexibilité et cette diversité des pratiques constituent une source de risque, compte tenu de l'incertitude qui pèse sur la qualité du service offert.

Les résultats de la recherche menée à Marrakech confirment ce risque. En termes d'apprentissage, les ONG enregistrent en moyenne des performances moins bonnes que les autres opérateurs d'alphabétisation (Programme général du MEN, programme des opérateurs publics). Les résultats de cette étude suggèrent également que cette performance moyenne est principalement induite par la vulnérabilité qui caractérise les formateurs mobilisés par les ONG.

Au-delà de leur portée spécifique liée à la nécessité de la stabilisation du corps des formateurs comme condition d'amélioration des performances des ONG, les résultats de l'étude suggèrent que l'apport indiscutable de ces dernières sur le plan de l'accès mérite d'être consolidé par des actions ciblant l'objectif de la qualité. Ceci passe par une volonté politique pour professionnaliser les activités de l'alphabétisation, en agissant de manière simultanée sur deux leviers :

- l'ajustement quantitatif et qualitatif des ressources mobilisées (inputs de l'opération d'alphabétisation) par rapport aux objectifs de réalisation fixés : ceci suppose l'amélioration de la quantité et surtout la qualité des inputs de l'opération de l'alphabétisation, aussi bien sur le plan des ressources humaines (formateurs, gestionnaire, superviseur) que sur le plan de la logistique (matériel didactique, conditions d'apprentissage...) ; bien que transversale à tous les programmes, cette nécessité d'amélioration de la qualité des inputs se pose avec plus d'acuité dans le cas du programme des ONG, où la diversité des pratiques est la règle dominante ;

- l'amélioration du pilotage du secteur par la mise en place d'un système de mesure, de suivi et d'évaluation, dont l'utilité est de permettre aux décideurs de faire des choix politiques mieux informés. Un tel système devrait intégrer la production et la diffusion de façon pérenne d'indicateurs sur la qualité et ses déterminants, la qualité étant mesurée en termes de niveau des apprentissages acquis, de leur durabilité, de leurs usages et leurs impacts. Ces indicateurs permettront de dresser une typologie des pratiques en termes d'efficience, qui servira de grille d'allocation des ressources mobilisées pour la politique de l'alphabétisation. Les pouvoirs publics peuvent, en toute connaissance de cause, allouer les moyens soit pour renforcer/généraliser les bonnes pratiques et/ou pour corriger, si cela s'avère opportun, les autres pratiques jugées inefficientes. 
La réalisation des objectifs de la politique d'alphabétisation passe par l'engagement politique de mettre en ouvre une politique volontariste de partenariat adossée à un système d'information pertinent. Le partenariat serait un levier pour mobiliser plus de ressources en adéquation avec les objectifs politiques fixés. Le système d'information, quant à lui, servirait à optimiser le pilotage en améliorant l'efficience de l'action. Si le partenariat avec la société civile ouvre des opportunités réelles pour des avancées substantielles en ce qui concerne l'accès, il reste insuffisant pour que ces avancées se traduisent par une élévation durable du niveau d'alphabétisme de la population ciblée. Les pouvoirs publics se doivent d'inscrire leur politique d'alphabétisation dans la durée, en l'adossant à un système d'information qui puisse rendre possible et endogène l'analyse des déterminants de la qualité qui servirait de base pour un pilotage basé sur l'efficience. Un tel système d'information devrait être construit par le mixage des dispositifs d'évaluation initiés au niveau central et par des dispositifs plus localisés/territorialisés initiés au niveau décentralisé, notamment par l'implication de l'Université et des structures décentralisées et déconcentrées des ministères concernés. C'est sans doute à ce prix que la politique d'alphabétisation pourrait s'inscrire dans un cercle vertueux et se hisser au premier rang des priorités éducatives qu'elle devrait occuper.

\section{BIBLIOGRAPHIE}

BOUGROUM M., IBOURK A., LOWENTHAL P. (2006) : La politique d'alphabétisation au Maroc: quel rôle pour le secteur associatif? Mondes en développement, 2006/2 $\mathrm{n}^{\circ} 134$, p. 63-77.

CERBELLE S. (2010): L'alphabétisation au Maroc, injonctions internationales, politique nationale et effets sur le terrain. Thèse de doctorat en sciences de l'éducation, Paris 5.

HCP (2004) : Recensement Général de la Population et de l'Habitat (RGPH). Rabat. COSEF (1999): Charte nationale d'éducation et de formation. Rabat, octobre.

SECAENF (2004) : Stratégie d'alphabétisation et d'éducation non formelle. Rabat, septembre.

UNESCO (2006): Rapport mondial sur le suivi EPT. L'alphabétisation, un enjeu mondial. Paris. 
